\title{
Learning curve or experience-related outcome: what really matters in paediatric laparoscopic pyeloplasty
}

\author{
Wojciech Panek ${ }^{1}$, Jakub Szmer ${ }^{2}$, Caroline F. Kuijper ${ }^{3}$, Rafal Chrzan ${ }^{2}$ \\ ${ }^{1}$ Department of Urology, $4^{\text {th }}$ Military Clinical Hospital, Wroclaw, Poland \\ ${ }^{2}$ Department of Paediatric Urology, Jagiellonian University Medical College, Krakow, Poland \\ ${ }^{3}$ Department of Paediatric Urology, Emma Children's Hospital, Amsterdam UMC, The Netherlands
}

Videosurgery Miniinv 2020; 15 (2): 377-381

DOI: https://doi.org/10.5114/wiitm.2019.89391

\begin{abstract}
Introduction: The process of improving one's skills over time is called a "learning curve". This term has attracted great attention during the last decades, especially in relation to laparoscopic techniques.

Aim: To assess the outcome of paediatric laparoscopic pyeloplasty (LP).

Material and methods: Retrospective analysis of the consecutive LPS. The inclusion criteria: (1) children aged $<18$ years, (2) transperitoneal approach, and (3) the same operating paediatric urologist (RC). Patients with a history of any procedure on the upper urinary tract were excluded. Any surgical reintervention during follow-up was defined as a failure. The outcomes of LPs performed before 2012 (G1) were compared to those conducted between 2012 and 2016 (G2). Fisher's exact test was used for statistical analysis.

Results: Ninety patients met the inclusion criteria, and a total of 95 LPS were performed. The mean operation time was $155 \mathrm{~min}$, and the mean hospitalisation period was 2.4 days. In G1, 19 patients underwent Anderson-Hynes LP, 16 had Fenger non-dismembered LP and two underwent vascular hitch. In G2, 54, 2 and 2 patients underwent these procedures, respectively. The overall success rate was $91.5 \%$. There were six failures in G1 and three in G2 $(p=0.147)$. Of the Anderson-Hynes LPs, $1 / 19$ in $G 1$ and $3 / 58$ in $G 2$ required reintervention $(p=1)$. For Fenger $L P s$, this was 4/16 and $0 / 2$, respectively $(p=1)$. Only one patient required reoperation after vascular hitch.

Conclusions: The surgeons' learning curve reflects their experience with regard to the entire therapeutic process, but not exclusively their manual skills.
\end{abstract}

Key words: pyeloplasty, laparoscopy, children, learning curve.

\section{Introduction}

Ureteropelvic junction obstruction (UPJO) is one of the most common pathologies of the urinary tract in children. Over the past few decades, the number of diagnosed but asymptomatic dilatations of the renal pelvis in young children has increased as a result of routine antenatal ultrasonography. However, hydronephrosis tends to decrease over time, and the majority of cases do not require surgical intervention [1, 2].
Clinical symptoms, progressive dilation of the collecting system and impaired renal function are all indications for pyeloplasty in the paediatric population [3]. Treatment of the UPJO means eliminating the obstruction, but how to achieve this goal depends on the surgeon's preference. Dismembered Anderson-Hynes pyeloplasty is considered to be the "gold standard" method, whereas non-dismembered techniques, such as V-Y pyeloplasty, Fenger pyeloplasty (longitudinal incision of the narrow part followed by

\section{Address for correspondence}

Rafal Chrzan MD, PhD, Department of Paediatric Urology, Jagiellonian University Medical College, 265 Wielicka St, 30-663 Krakow, Poland, phone: +48 1233390 55, e-mail: rafal.chrzan@uj.edu.pl 
its transverse closure) or Hellstrom vascular hitch (relocation of the lower pole crossing vessel), are less popular [4-6]. Each of these procedures can be performed with either open or minimally invasive techniques (laparoscopic or robotic-assisted). The overall success rate of pyeloplasty varies between $81 \%$ and $100 \%[5,7]$. The accepted failure rate for an Anderson-Hynes dismembered laparoscopic pyeloplasty (LP) is approximately $5 \%$ [8-11].

Although many independent factors may influence the final outcome, the personal experience of the operating surgeon is most likely to be of great importance, especially when the laparoscopic technique is used. The process of improving one's skills over time, in this case by performing an increasing number of procedures, is called a "learning curve" [12]. For the patients it means fewer failures.

\section{Aim}

The goal of this study was to evaluate the outcomes of LP in terms of a surgeon's learning curve, and to determine whether establishing a uniform policy for the treatment of UPJO leads to a better overall outcome in paediatric patients.

\section{Material and methods}

This study involved a retrospective analysis of LPS performed between 2006 and 2016. The inclusion criteria for this study were as follows: 1) age between 0 and 18 years, 2) laparoscopic transperitoneal approach, and 3) the same operating surgeon. Patients who had undergone any previous procedures on the upper urinary tract and those who dropped out from follow-up were excluded. In one case, conversion was needed, so this patient was also excluded from the series.

The follow-up period was longer than 1 year in all patients. All patients underwent regular physical examinations, with an ultrasound performed 6-8 weeks and again at 4 and 12 months after surgery. Our policy is to repeat a diuretic scintigraphy only in symptomatic patients or those with suspicion of recurrence due to persistent dilation of the collecting system.

Any surgical re-intervention at the ureteropelvic junction (UPJ) level (open or endoscopic) was classified as a failure, which represented the end-point of the study. Fisher's exact test was used for the statistical analysis.
The first LP was performed in 2006, and various surgical methods have been used since then in our institutions. Between 2006 and 2011, the decision of which technique to apply for each case was made intraoperatively. No strict criteria were defined at that time, but a non-dismembered LP was often used for two reasons: 1) to avoid damaging the blood supply of the upper ureter, and 2) to reduce the need for intracorporeal suturing/handling. Crossing vessels (CVs) were approached by either mobilisation, fixation to the pelvis (the Hellstrom procedure) or by dorsal transposition during a dismembered LP. From the beginning of 2012, Anderson-Hynes dismembered pyeloplasty became the leading procedure, with dorsal transposition of CVs performed if found intraoperatively. The other procedures were only used in selected cases. This change in our protocol was introduced as a result of an internal review of the outcomes and personal communication with other experienced laparoscopists. Hence, to answer the study question, the cohort was divided into two groups: $\mathrm{G} 1$, including all consecutive patients operated on between 2006 and 2011; and G2, all consecutive patients operated on between 2012 and 2016 (predominantly using Anderson-Hynes LP).

In total, 94 patients underwent LP between 2006 and 2016. Of these, four patients were excluded: one patient had undergone a previous surgical intervention due to duplication of the collecting systems, two procedures were performed by other paediatric urologists, and a conversion was needed in 1 patient. In 5 patients, a bilateral procedure was performed.

\section{Results}

The total number of LPs was 95, performed in 34 females and 56 males. The mean patient age was 9 years and 5 months. Fifty-seven were done on the left side $(60 \%)$. The mean operating time was 155 min (range: 88-350 min), including the bilateral cases, and the mean hospital stay after surgery was 2.4 days (range: $1-10$ days). CVs were found in $57 / 95$ cases, which were treated by means of dorsal transposition in combination with the Anderson-Hynes LP $(n=46)$, translocation in combination with the Fenger LP $(n=5)$ or by the Hellstrom vascular hitch $(n=6)$. Patient data are shown in Table I.

The overall failure rate was $9.5 \%$. The most successful method was the Anderson-Hynes procedure (Table II). 
Table I. Surgery data for patients in groups 1 and 2

\begin{tabular}{|lccc|}
\hline Parameter & G1 & G2 & Total \\
\hline Number of LPs & 37 & 58 & 95 \\
\hline AH procedure & 19 & 54 & 73 \\
\hline Fenger procedure & 16 & 2 & 18 \\
\hline Hellstrom procedure & 2 & 2 & 4 \\
\hline CVs & 16 & 41 & 57 \\
\hline
\end{tabular}

AH - Anderson-Hynes, CVs - crossing vessels, G1 - group 1, G2 - group 2, $L P$ - laparoscopic pyeloplasty.

In G1, 6 patients required re-do surgery: four after Fenger LPs (25\% of all Fenger procedures during this first period), one after Anderson-Hynes LP (5.3\% of all Anderson-Hynes procedures during this period), and one after the Hellstrom procedure $(50 \%$ of all Hellstrom procedures during this period). In $\mathrm{G} 2$, three (5.1\%) surgical re-interventions were needed, all of which were after an Anderson-Hynes LP. The details are presented in Table III. The differences between the groups were not statistically significant (Table IV).

\section{Discussion}

Different aspects of the "learning curve" can be taken into consideration in relation to any surgical method (e.g. the duration of the procedure) but for the patient the final outcome counts.
Table II. Outcome of laparoscopic pyeloplasty

\begin{tabular}{|lccc|}
\hline Total & $\begin{array}{c}\text { Total } \\
\text { number }\end{array}$ & $\begin{array}{c}\text { Successful } \\
\text { cases }\end{array}$ & $\begin{array}{c}\text { Failure rate } \\
(\%)\end{array}$ \\
\hline Anderson-Hynes & 73 & 69 & 5.5 \\
\hline Fenger & 18 & 14 & 22.2 \\
\hline Hellstrom & 4 & 3 & 25 \\
\hline Total & 95 & 86 & 9.5 \\
\hline
\end{tabular}

In our cohort, although the difference was not statistically significant ( $p=0.147)$, there were definitely more failures in the first period (16.2\%) than in the second period (5.1\%). Based on those results, one could draw the conclusion that there is a clear learning curve for LP in the paediatric population. There was an obvious improvement over time, and the overall success rate in $\mathrm{G} 2$ is similar to the accepted numbers in the literature [13].

The Anderson-Hynes method is considered the gold standard procedure for the treatment of UPJO [3]. Valla et al. as well as many others showed that both open and minimally invasive techniques are highly effective in children, with failure rates of less than $5 \%[4,5,14]$. In our cohort, the results of the Anderson-Hynes LP were similar in both groups (G1 and $\mathrm{G} 2$ ), and are comparable to those data reported by previous studies. Thus, considering only this particular method, one can state that there is no learning curve effect for LP in children.

Table III. Results in group 1 (G1) and group 2 (G2)

\begin{tabular}{|c|c|c|c|c|c|c|}
\hline \multirow[t]{2}{*}{ Parameter } & \multicolumn{3}{|c|}{$\mathrm{G} 1$} & \multicolumn{3}{|c|}{$\mathrm{G} 2$} \\
\hline & $N$ & Success & Failure & $N$ & Success & Failure \\
\hline All LPS & 37 & 31 & 6 & 58 & 55 & 3 \\
\hline Anderson-Hynes & 19 & 18 & 1 & 54 & 51 & 3 \\
\hline Fenger & 16 & 12 & 4 & 2 & 2 & 0 \\
\hline Hellstrom & 2 & 1 & 1 & 2 & 2 & 0 \\
\hline
\end{tabular}

LPS - laparoscopic pyeloplasties.

Table IV. Statistical analysis for laparoscopic pyeloplasty

\begin{tabular}{|lcccc|}
\hline Parameter & G1 overall & G2 overall & G1 A-H & 19 \\
\hline Number of patients & 37 & 58 & 54 \\
\hline Failure rate $(\%)$ & 16.2 & 5.1 & 5.26 & 5.55 \\
\hline P-value score & & 0.147 & & 1 \\
\hline
\end{tabular}

G1-group 1, G2-group 2, AH-Anderson-Hynes. 
The Fenger and Y-V pyeloplasties, as well as the vascular hitch procedure, are less popular due to their uncertain and diverse outcomes. Szydetko et al. showed that non-dismembered LP is highly effective in adults. In other reports that compared the efficacy of dismembered and non-dismembered pyeloplasties, a non-significant or only a slight difference between those techniques has been demonstrated in adults $[15,16]$. Literature on the results of non-dismembered pyeloplasty in children is sparse. Polok et al. reported that the success rate of the Fenger procedure was $96 \%$ with an open approach and 85\% for the laparoscopic approach. [17]. Only a few other small series have been published on this topic over the past few years [6, 18]. The Hellstrom method, also known as the vascular hitch procedure, has been abandoned for decades, but has been rediscovered thanks to the increasing popularity of laparoscopic approaches. Chiarenza et al. presented their results, with an almost $100 \%$ success rate, and Madec et al. achieved similar outcomes $[19,20]$. In our cohort, the success rate of the Fenger procedure was $75 \%$ in G1 and $100 \%$ in $\mathrm{G} 2$, and for the Hellstrom method it was $50 \%$ and $100 \%$, respectively. In our hands, these methods were clearly less effective, but there was improvement over time. At this point, an interesting issue comes to light. During the first period, 18 procedures other than Anderson-Hynes pyeloplasty were performed, of which five required re-intervention. During the second period, there were only four procedures other than Anderson-Hynes pyeloplasty, and none of them required surgical re-intervention. The sample size is too small to show any significant difference, but it is possible that reserving the alternative methods only for selected cases may improve the overall outcome. Hence, this raises the question of the proper indications and surgical performance.

The goal of any surgical intervention is to achieve the best possible result. It has been demonstrated that good outcomes are related to the number of procedures conducted by the surgeon $[12,21]$. This relationship is called a learning curve, and this term attracts a lot of attention in the medical literature. Surgeons who are over their learning curve have reached the optimal level for that particular method. The outcome of the procedure improves over time, but this is just a derivate of surgical skill. The learning curve is only interesting for the patient when he/ she becomes free of symptoms without complica- tions. This can be achieved when the whole process proceeds correctly, which includes making the right diagnosis, defining the right indications for intervention, and choosing the best treatment method. In this way, the patient receives personalised treatment, and the learning curve related to the particular method is a part of this course. Hence, we believe that an "experience-related learning curve" is more appropriate.

There are hardly any recommendations for this combination of insight and technique; however, the majority of surgeons try out several techniques before they choose the one that fits both the patient's status and their own surgical skills. To our knowledge, this is the first paper to analyse the learning curve in this way.

The obvious limitation of this study is the small number of patients. Furthermore, this study is retrospective, and is based on only a single surgeon's experience. Hence, further analysis of a similar cohort from another centre would be favourable.

\section{Conclusions}

Critical internal analysis is essential to improve the overall outcomes of LP. The surgeons' learning curve reflects their experience with regard to the entire therapeutic process, but not their manual skills.

\section{Conflict of interest}

The authors declare no conflict of interest.

\section{References}

1. Fefer S, Ellsworth P. Prenatal hydronephrosis. Pediatr Clin North Am 2006; 53: 429-47.

2. Thomas DFM. Prenatal diagnosis: what do we know of longterm outcomes? J Pediatr Urol 2010; 6: 204-11.

3. Gopal M, Peycelon M, Caldamone A, et al. Management of ureteropelvic junction obstruction in children - a roundtable discussion. J Pediatr Urol 2019; pii: S1477-5131(19)30133-0. doi: 10.1016/j.jpurol.2019.05.010.

4. Tan BJ, Smith AD. Ureteropelvic junction obstruction repair: when, how, what? Curr Opin Urol 2004; 14: 55-9.

5. Sukumar S, Roghmann F, Sood A, et al. Correction of ureteropelvic junction obstruction in children: national trends and comparative effectiveness in operative outcomes. J Endourol 2014; 28: 592-8.

6. Amón Sesmero JH, Delgado MC, de la Cruz Martín B, et al. Laparoscopic pyeloplasty: always dismembered? J Endourol 2016; 30: 778-82.

7. Romao RLP, Koyle MA, Pippi Salle JL, et al. Failed pyeloplasty in children: revisiting the unknown. Urology 2013; 82: 1145-9. 
8. Yeung CK, Tam YH, Sihoe JD, et al. Retroperitoneoscopic dismembered pyeloplasty for pelvi-ureteric junction obstruction in infants and children. BJU Int 2001; 87: 509-13.

9. van der Toorn F, van den Hoek J, Wolffenbuttel KP, et al. Laparoscopic transperitoneal pyeloplasty in children from age of 3 years: our clinical outcomes compared with open surgery. J Pediatr Urol 2013; 9: 161-8.

10. García-Aparicio L, Blazquez-Gomez E, Martin O, et al. Anderson-Hynes pyeloplasty in patients less than 12 months old. Is the laparoscopic approach safe and feasible? J Endourol 2014; 28: 906-8.

11. Autorino R, Eden C, El-Ghoneimi A, et al. Robot-assisted and laparoscopic repair of ureteropelvic junction obstruction: a systematic review and meta-analysis. Eur Urol 2014; 65: 430-52.

12. Hopper AN, Jamison MH, Lewis WG. Learning curves in surgical practice. Postgrad Med J 2007; 83: 777-9.

13. Seixas-Mikelus SA, Jenkins LC, Williot P, et al. Pediatric pyeloplasty: comparison of literature meta-analysis of laparoscopic and open techniques with open surgery at a single institution. J Urol 2009; 182: 2428-32.

14. Valla JS, Breaud J, Griffin SJ, et al. Retroperitoneoscopic vs open dismembered pyeloplasty for ureteropelvic junction obstruc tion in children. J Pediatr Urol 2009; 5: 368-73.

15. Szydetko T, Kasprzak J, Lewandowski J, et al. Dismembered laparoscopic Anderson-Hynes pyeloplasty versus nondismembered laparoscopic $\mathrm{Y}-\mathrm{V}$ pyeloplasty in the treatment of patients with primary ureteropelvic junction obstruction: a prospective study. J Endourol 2012; 26: 1165-70.

16. Jarrett TW, Chan DY, Charambura TC, et al. Laparoscopic pyeloplasty: the first 100 cases. J Urol 2002; 167: 1253-6.

17. Polok M, Chrzan R, Veenboer P, et al. Nondismembered pyeloplasty in a pediatric population: results of 34 open and laparoscopic procedures. Urology 2011; 78: 891-4.

18. Casale P, Grady RW, Joyner BD, et al. Comparison of dismembered and nondismembered laparoscopic pyeloplasty in the pediatric patient. J Endourol 2004; 18: 875-8.

19. Chiarenza SF, Bleve C, Fasoli L, et al. Ureteropelvic junction obstruction in children by polar vessels. Is laparoscopic vascular hitching procedure a good solution? Single center experience on 35 consecutive patients. J Pediatr Surg 2016; 51: 310-4.

20. Madec FX, Faraj S, Villemagne T, et al. Laparoscopic transposition of lower-pole crossing vessels: long-term follow-up of 33 patients at puberty. J Pediatr Urol 2016; 12: 226.e1-6.

21. Sukumar S, Djahangirian O, Sood A, et al. Minimally invasive vs open pyeloplasty in children: the differential effect of procedure volume on operative outcomes. Urology 2014; 84: 180-4.

Received: 20.09.2019, accepted: 4.10.2019. 